\title{
Comportamiento térmico en ríos mediterráneos andinos de la zona centro-sur de Chile
}

\author{
Pablo Pedreros ${ }^{1, *}$, Meyer Guevara ${ }^{1}$, Ricardo Figueroa ${ }^{1}$, Alberto Araneda ${ }^{1}$, Alejandra Stehr ${ }^{1}$, \\ Oscar Link ${ }^{2}$ y Roberto Urrutia ${ }^{1}$
}

${ }^{1}$ Centro de Ciencias Ambientales EULA-Chile, Universidad de Concepción, Casilla 160-C, Concepción, Chile.

${ }^{2}$ Departamento de Ingeniería Civil, Universidad de Concepción, Casilla 160-C, Concepción, Chile.

* Autor responsable de la correspondencia: papedrer@gmail.com

Recibido: 5/7/12

Aceptado: $21 / 2 / 13$

\begin{abstract}
Thermal behavior of Mediterranean Andean streams in South-Central Chile

Despite of the importance of temperature in the fluvial ecosystems, the knowledge of its spatial and temporal variability in Andean rivers is limited, thus it is necessary to clarify the relative importance of site-specific factors in controlling the temperature of rivers. The aim of this research was to characterize the thermal regime of five Andean streams through an altitudinal gradient. The results show that there is a spatial and temporal variability in water temperature with a relatively rapid rise in temperature in mid-December, being stable in January and February, and a rapid decrease at the beginning of March and April. The thermal heterogeneity recorded in the high Andean zone of the Biobio River Basin makes it clear that geomorphology characteristics of each site are important in regulating water temperature, associated mainly with the altitude and shade; this latter mainly generated by high mountains and in some cases by the timberline. It is known that thermal variability, mainly the maximum and minimum mean temperatures; causing severe stress on stenotherm organisms. Therefore, a deeper knowledge of the river temperature is essential for the management and future protection of the Andean freshwater ecosystems to mitigate the impacts associated to the global warming.
\end{abstract}

Key words: Local factors, altitudinal pattern, thermal regime, Andean streams.

\section{RESUMEN}

\section{Comportamiento térmico en ríos mediterráneos andinos de la zona centro-sur de Chile}

A pesar de la importancia que tiene la temperatura en los sistemas fluviales, el conocimiento de su variabilidad espaciotemporal en ríos de Chile es limitado. Tales estudios son necesarios para aclarar la importancia relativa de los factores locales a la hora de controlar la temperatura de los ríos. La presente investigación tuvo por objetivo caracterizar el régimen térmico de cinco ríos andinos de la región del Biobío a través de un gradiente altitudinal. Los resultados muestran que existe una variabilidad espacio-temporal en la temperatura del agua con un incremento relativamente rápido de la temperatura a mediados de diciembre, manteniéndose en enero y febrero, para disminuir rápidamente a principio de marzo y abril. La heterogeneidad térmica encontrada en la zona andina de la cuenca del río Biobío deja de manifiesto que las características geomorfológicas de cada sitio son relevantes en la regulación de la temperatura del agua, asociada principalmente a la altitud y sombra, esta última generada principalmente por las altas montañas y en algunos casos por la vegetación arbórea.

Se prevé que cambios principalmente en las temperaturas medias máximas y mínimas podrían ocasionar un severo estrés en organismos estenotermos. Por lo tanto, un conocimiento detallado de la temperatura en ríos andinos podría proveer de información necesaria para direccionar la mitigación de los impactos asociados al calentamiento global.

Palabras clave: Factores locales, patrón altitudinal, régimen térmico, ríos andinos. 


\section{INTRODUCCIÓN}

La temperatura es una de las variables del hábitat físico más importantes en los ecosistemas fluviales, debido a que afecta la respuesta ecológica funcional y estructural de los organismos acuáticos (Vannote \& Sweeney, 1980; Hawkins et al., 1997; Jacobsen et al., 1997), así como las reacciones fisicoquímicas que ocurren en dichos sistemas (Berner \& Berner, 1996; Webb, 1996; Erickson \& Stefan, 2000). No obstante, el conocimiento ligado a la variabilidad térmica natural de los ríos es limitado, dado el nivel de alteración que presentan estos sistemas (Malmqvist $\&$ Rundle, 2002). Además de esto, se proyecta que la temperatura superficial del planeta seguirá aumentando como resultado del calentamiento global $\left(3-5^{\circ} \mathrm{C}\right.$ durante los próximos años; IPCC, 2007), lo cual condicionaría no solo los regímenes térmicos estacionales, sino también la respuesta ecológica de los sistemas fluviales, principalmente la distribución de las especies dulceacuícolas (Heino, 2002; Caissie, 2006; Brown et al., 2007).

En condiciones naturales, el factor climático es el principal modelador de la temperatura en ríos, sobre todo aquellos parámetros que presentan una variabilidad interanual (p. ej. estacionalidad del sol, temperatura del aire, viento y humedad relativa; Malcolm et al., 2004). Sin embargo, también existen factores locales (p. ej. vegetación de ribera, aguas subterráneas) que contribuyen a la heterogeneidad térmica, los cuales han sido identificados por Alexander \& Caissie (2003), Johnson (2004), Royer \& Minshall (1997) y Poff $\&$ Ward (1990). Estos factores raramente influyen de forma independiente en la temperatura, por lo que su importancia es un desafío, debido a que pueden variar en forma diaria, estacional, así como espacialmente. Estudios desarrollados durante las últimas décadas han permitido constatar que la radiación solar de onda corta y la temperatura del aire son los reguladores primarios de la temperatura en los sistemas fluviales (Sinokrot \& Stefan, 1994; Webb \& Zhang, 1997; Evans et al., 1998). Alexander \& Caissie (2003) demuestran que el intercambio de calor entre la atmósfera y la superficie del agua explica gran parte de las fluctuaciones térmicas diarias en ríos someros. Sin embargo, la naturaleza del intercambio energético puede variar significativamente de acuerdo con las características geomorfológicas del río (p. ej. rápidos, rápidos-someros, pozas; Webb et al., 2008).

En Chile, la diversidad geológica y continental conforma una realidad territorial latitudinalaltitudinal de tal magnitud, que permite diferenciar térmicamente una variedad de ecosistemas acuáticos. Sin embargo, los estudios del régimen térmico son muy limitados, conociéndose solo los de Link et al. (2012), Monsalve et al. (2012) y Link et al. (2009). Esto genera una escasez de información sobre la dinámica y heterogeneidad térmica de los ríos en condiciones naturales. En este contexto, los ríos alto-andinos se presentan como unidades ecológicas favorables para estudiar los patrones térmicos, ya que a menudo se encuentran escasamente alterados y presentan un gradiente térmico que depende marcadamente de las condiciones ambientales locales y la fuente que los alimenta (p. ej. glaciar, subterránea y/o termal). Ambos factores son importantes para moderar las temperaturas (Poole \& Berman, 2001; Caissie, 2006), en particular las altas temperaturas estivales, que aumentarían como consecuencia del calentamiento global (Falvey \& Garreaud, 2009), siendo limitante para la distribución y supervivencia futura de ciertas especies acuáticas (p. ej. Plecoptera; Palma \& Figueroa, 2008) de las zonas andinas. De acuerdo con lo planteado anteriormente, el objetivo del presente trabajo es caracterizar el régimen térmico de cinco ríos alto-andinos de la región del Biobío a través de un gradiente altitudinal, para identificar los factores locales que afectan la temperatura de los ríos.

\section{MATERIAL Y MÉTODOS}

\section{Área de estudio}

La zona de estudio se localizó entre los 37.74$38.08^{\circ} \mathrm{S} ; 71.39-71.14^{\circ} \mathrm{W}$ y consideró cinco ríos alto-andinos de la cuenca del río Biobío: Lomín, Chaquilvín, Quepuca, Pangue y Queuco (Fig. 1). Todos los sitios presentan características climáticas típicas de ríos mediterráneos (Gasith \& Resh, 1999). Se describe el régimen hidrológico de 
todos los sitios como pluvio-nival, con pico de máximo caudal en invierno y primavera. El sedimento de la zona de estudio está constituido principalmente por un conglomerado de roca ígnea con intrusiones sedimentarias, asociado fundamentalmente a las características fluvio-glacio-volcánicas de la zona (Mardones et al., 1992). El sustrato acuático consiste en una mezcla de cantos rodados, piedras, grava y arena, mientras que la vegetación está constituida por una mezcla entre bosque nativo perenne (p. ej. Drymis winteri), caducifolio (Nothophagus spp.), matorral y estepa andina (Hajek, 1991; Dallman,
1998). La figura 1 muestra el uso de suelo de cada una de las microcuencas estudiadas. Los tramos de río fueron clasificados de acuerdo con sus diferencias de altitud: ríos de mayor altura (> $800 \mathrm{msnm}$ ) con mezcla de matorral y estepa alto-andina; de altitud media (500-800 msnm) con bosque achaparrado y renoval nativo, y de baja altitud $(<500 \mathrm{msnm})$ con mezcla de bosque y renoval nativo. El renoval nativo corresponde al bosque nativo secundario originado por semillas y/o reproducción vegetativa después de una perturbación antrópica o natural. Cada sitio de estudio fue caracterizado incluyendo

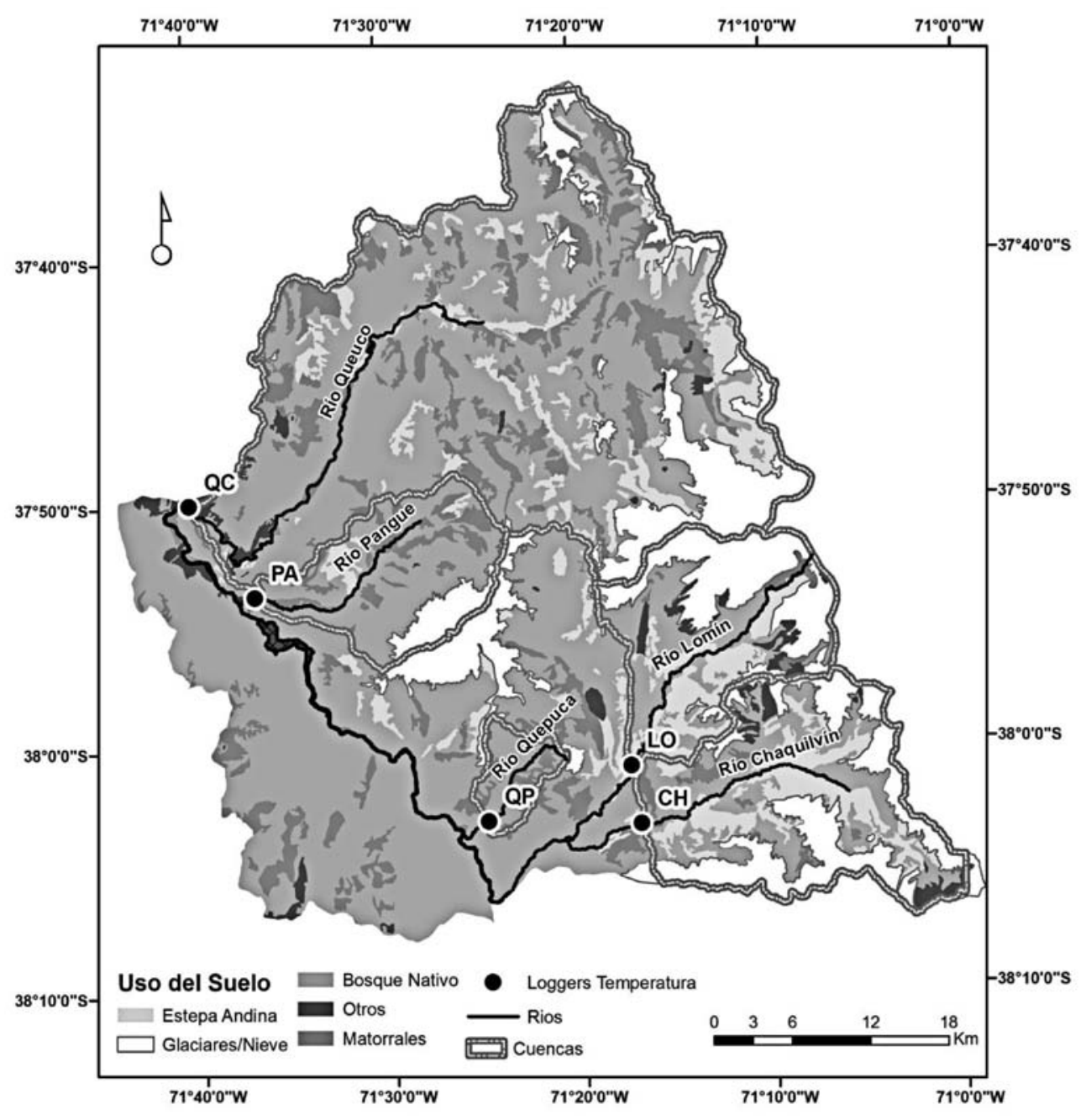

Figura 1. Localización de los sitios de estudio en el sector andino de la cuenca del río Biobío. Location of study sites in the Andean zone of the Biobio River basin. 
Tabla 1. Caracterización física de los cinco ríos andinos estudiados desde julio de 2010 a junio de 2011 . LO = Lomín; CH = Chaquilvín; QP = Quepuca; PA = Pangue y QC = Queuco. Physical characterization of the five Andean rivers studied during July 2010 and June 2011. LO = Lomín; $C H=$ Chaquilvín; $Q P=$ Quepuca; $P A=$ Pangue y $Q C=Q$ Quco.

\begin{tabular}{|c|c|c|c|c|c|}
\hline Sitio & LO & $\mathrm{CH}$ & QP & PA & QC \\
\hline \multirow{2}{*}{ Localización } & $38^{\circ} 00^{\prime} 57.24^{\prime \prime} \mathrm{S}$ & $38^{\circ} 03^{\prime} 21.5^{\prime \prime} \mathrm{S}$ & $38^{\circ} 03^{\prime} 8.05^{\prime \prime} \mathrm{S}$ & $37^{\circ} 53^{\prime} 45.04^{\prime \prime} \mathrm{S}$ & $37^{\circ} 49^{\prime} 56.9^{\prime \prime} \mathrm{S}$ \\
\hline & $71^{\circ} 17^{\prime} 24.02^{\prime \prime} \mathrm{W}$ & $71^{\circ} 16^{\prime} 52.29^{\prime \prime} \mathrm{W}$ & $71^{\circ} 24^{\prime} 57.22^{\prime \prime} \mathrm{W}$ & $71^{\circ} 36^{\prime} 51.79^{\prime \prime} \mathrm{W}$ & $71^{\circ} 40^{\prime} 12.54^{\prime \prime} \mathrm{W}$ \\
\hline Altitud (msnm) & 936 & 882 & 750 & 475 & 370 \\
\hline Área de la cuenca $\left(\mathrm{km}^{2}\right)$ & 214.52 & 298.92 & 35.32 & 156.03 & 983.61 \\
\hline Aspecto & $\mathrm{E}$ & $\mathrm{NE}$ & NE & NO & $\mathrm{E}$ \\
\hline Ancho máximo del río (m) & $8-12$ & $18-25$ & $5-7$ & $7-13$ & $45-65$ \\
\hline Profundidad (m) & $0.20-0.40$ & $0.21-0.50$ & $0.15-0.37$ & $0.22-0.45$ & $0.20-0.51$ \\
\hline Orden & 2 & 3 & 2 & 3 & 3 \\
\hline Distancia desde el origen $(\mathrm{km})$ & 25.919 & 19.737 & 10.827 & 17.827 & 71.858 \\
\hline
\end{tabular}

datos geográficos, además de características físicas del río (Tabla 1).

\section{Recolección de datos}

La temperatura del agua y del aire fue medida continuamente utilizando registradores de datos marca HOBO, modelo UA-001-08 ( $-20^{\circ}$ a $70{ }^{\circ} \mathrm{C}$ ), con una precisión de $\pm 0.5^{\circ} \mathrm{C}$. Estos fueron programados mediante el software HOBOware para registrar y almacenar la temperatura a intervalos de $15 \mathrm{~min}$; además se calibraron durante 24 horas previamente a su instalación en cada sitio de estudio, siguiendo la metodología basada en Haidekker \& Hering, 2008. Los registradores se introdujeron en tubos de PVC (diámetro $=35 \mathrm{~mm}$; longitud $=15 \mathrm{~cm}$ ) a fin de evitar que recibieran radiación directa que alterara la temperatura del sensor. El área de drenaje, la distancia desde el origen y el orden del río de cada sitio de muestreo fueron obtenidos de las bases de datos cartográficas del IGM (Instituto Geográfico Militar; 1:50 000), mientras que la ubicación geográfica y la altitud (msnm) se determinaron utilizando un GPS Garmin Xtrex. El ancho y la profundidad media se estimaron in situ en cada lugar de muestreo. La velocidad de la corriente se midió con un velocímetro digital Flow Probe, modelo FP111.

\section{Análisis estadístico}

La evaluación de heterogeneidad térmica se basó en los registros efectuados en los cinco ríos desde julio de 2010 a junio de 2011, a excepción del río Queuco, donde la recogida de datos comenzó en agosto 2010. Para cada sitio de estudio se determinaron cuatro variables térmicas: (i) temperatura media diaria, (ii) temperatura máxima diaria, (iii) temperatura mínima diaria, y (iv) rango térmico diario, el cual fue calculado como la diferencia entre la temperatura máxima y mínima diaria ( $\lambda$ media diaria). Además, se estimó la tasa media de calentamiento y de enfriamiento en primavera y otoño $\left({ }^{\circ} \mathrm{C}\right.$ día ${ }^{-1}$ ) (Uehlinger et $a l ., 2003)$, ambas obtenidas de la regresión lineal de la temperatura media diaria en el período de tiempo 21 septiembre-21 diciembre y 21 marzo21 junio, respectivamente. Paralelamente, se determinó la cantidad de horas que cada sitio recibe de radiación solar directa, a partir de la relación entre el ángulo cenital y el efecto de sombra generado por las altas montañas, siguiendo la metodología propuesta por Tung et al. (2006).

Se utilizó el análisis de varianza de un factor (ANOVA) con test de Tuckey post hoc para establecer diferencias en las variables térmicas (temperatura media diaria y rango térmico) y la estacionalidad entre los sitios y épocas de estudio. Las correlaciones y modelos de regresión se consideraron significativos cuando $p<0.05$. Como una medida de asociación entre la temperatura del aire y la del agua, se estimó la correlación de Pearson $(r)$ para cada sitio de estudio. Finalmente, se determinaron los grados-día para el período julio 2010-junio 2011 (Arscott et al., 2001). Este parámetro fue calculado a escala mensual y anual a partir de la suma de las temperaturas me- 
dias diarias por encima de $0{ }^{\circ} \mathrm{C}$, estableciendo la influencia de la energía térmica disponible en los organismos acuáticos.

\section{RESULTADOS}

La temperatura media diaria del aire presentó un patrón sinusoidal con una media diaria máxima, ocurrida el 23 de enero, que fluctuó entre $21.52{ }^{\circ} \mathrm{C}$ (río Quepuca) y $26.47^{\circ} \mathrm{C}$ (río Queuco), y una mínima, el 22 de julio, que fluctuó entre
$-6.32{ }^{\circ} \mathrm{C}$ (río Chaquilvín) y $0.88^{\circ} \mathrm{C}$ (río Queuco) (Fig. 2). La variabilidad media anual de la temperatura del aire entre los sitios con mayor y menor altitud fluctuó entre 9.36 y $12.49^{\circ} \mathrm{C}$, respectivamente. La comparación en esta microescala temporal (julio de 2010 a junio de 2011) reveló que a altitudes bajas las temperaturas son más cálidas, mientras que a altitudes media-altas los patrones térmicos del aire son más fríos y similares entre sí.

La temperatura del agua muestra un patrón sinusoidal similar al registrado en la temperatura del aire (véanse Fig. 2 y Tabla 2). En gene-
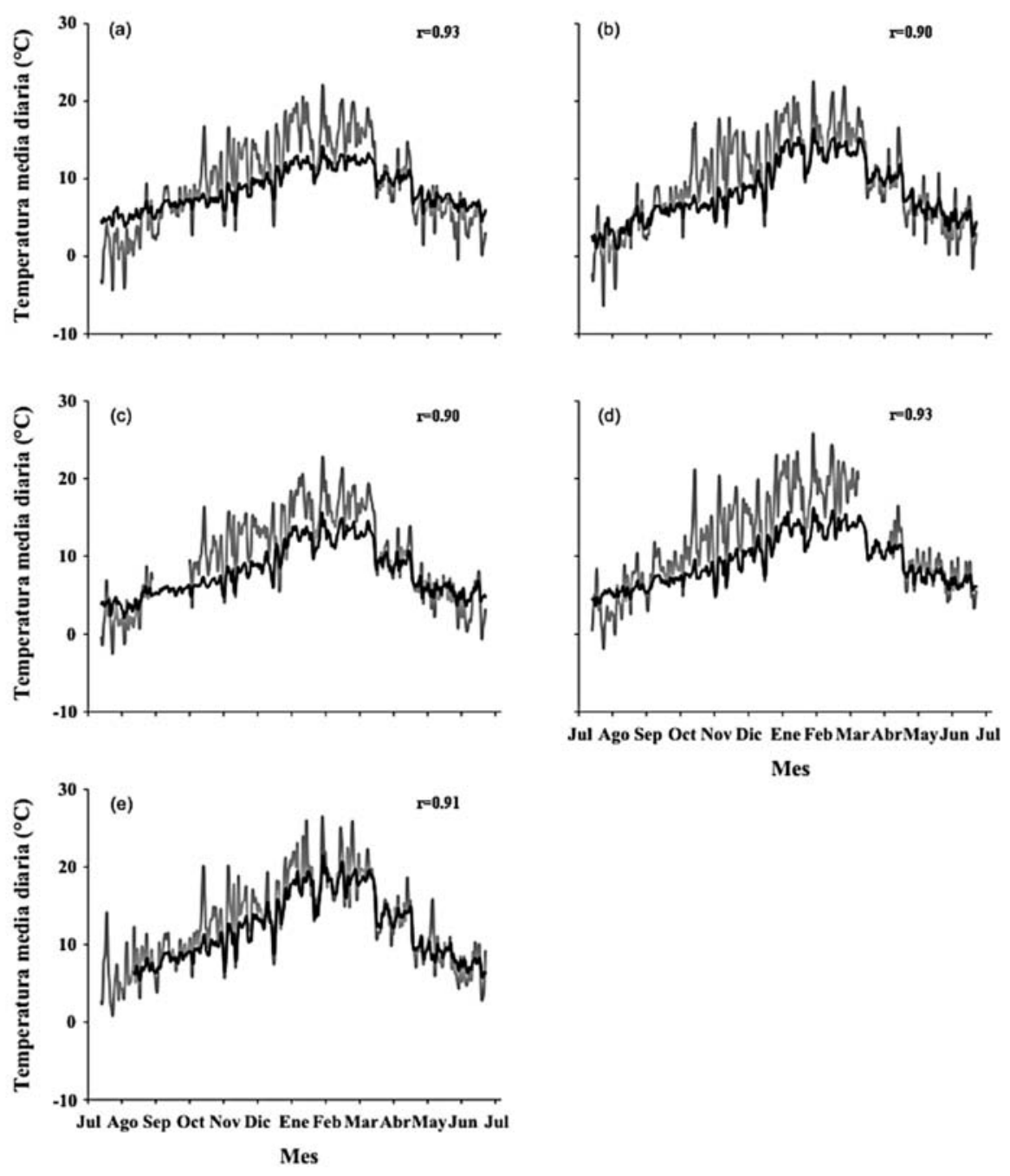

Figura 2. Temperatura media diaria del aire (línea clara) y agua (línea oscura) durante un ciclo anual de los ríos (a) Lomín, (b) Chaquilvín, (c) Quepuca, (d) Pangue y (e) Queuco. Daily mean air (light line) and water (dark line) temperature during an annual cycle in the (a) Lomín, (b) Chaquilvín, (c) Quepuca, (d) Pangue, (e) Queuco streams. 
Tabla 2. Características térmicas del agua $\left({ }^{\circ} \mathrm{C}\right)$ y horas de radiación directa ( \% RD) reportadas durante el ciclo anual julio de 2010 a junio de 2011, en ríos mediterráneos alto-andinos del centro-sur de Chile. Thermal characteristics of water $\left({ }^{\circ} \mathrm{C}\right)$ and hours of direct radiation (\% DR) reported during an annual cycle, from July 20120 to June 2011, in Andean Mediterranean rivers in South-Central Chile.

\begin{tabular}{|c|c|c|c|c|c|c|c|c|c|c|c|}
\hline Río & Altitud & $\mathbf{T}_{\text {media anual }}$ & $\mathbf{T}_{\text {media mensual }}$ & $\mathbf{T}_{\text {media diaria }}$ & $\lambda_{\text {media diaria }}$ & $\mathbf{T}_{\text {máx }}$ & Mes & $\mathbf{T}_{\text {mín }}$ & Mes & Grados-día & $\% \mathrm{RD}$ \\
\hline Lomín & 936 & 8.07 & $5.01-12.16$ & $3.78-14.04$ & $0.51-7.08$ & 16.90 & Enero & 2.41 & Agosto & 2976 & 40.02 \\
\hline Chaquilvín & 882 & 7.53 & $2.82-13.65$ & $0.97-16.32$ & $0.73-9.63$ & 19.28 & Enero & 0.12 & Julio & 2822 & 46.63 \\
\hline Quepuca & 750 & 7.96 & $3.96-12.95$ & $2.14-15.56$ & $0.51-8.49$ & 19.28 & Enero & 1.00 & Agosto & 2716 & 42.85 \\
\hline Pangue & 475 & 8.85 & $4.93-13.88$ & $3.52-15.91$ & $0.40-8.60$ & 20.04 & Enero & 2.73 & Julio & 3234 & 45.78 \\
\hline Queuco & 370 & 11.87 & $5.43-21.49$ & $6.84-18.20$ & $0.20-6.78$ & 24.54 & Enero & 1.87 & Julio & 3763 & 40.30 \\
\hline
\end{tabular}

ral, los registros térmicos de los cinco ríos altoandinos estuvieron caracterizados por un incremento relativamente rápido de la temperatura a mediados de diciembre, manteniéndose en enero y febrero, para disminuir rápidamente a principio de marzo y abril. Llama la atención que el río Pangue $(475 \mathrm{msnm})$ presentó un patrón térmico similar a los ríos de mayor altitud, Lomín (936 msnm) y Chaquilvín (882 msnm), y distinto al del río Queuco (370 $\mathrm{msnm}$ ), de menor altitud. Esto podría deberse a que el río Pangue tiene afluentes importantes de origen glaciar, mientras que el Queuco se nutre de la escorrentía superficial y aportes subterráneos. Las temperaturas fueron máximas en enero para todos los sitios de estudio, mientras que las mínimas fueron en julio (Chaquilvín, Pangue y Queuco) y agosto (Lomín y Quepuca). En cuanto al rango térmico, este tuvo su mayor amplitud en el río Chaquilvín, con valores entre $0.73-9.63^{\circ} \mathrm{C}$, mientras que el río Queuco presentó la menor amplitud, con valores entre $0.20-6.78^{\circ} \mathrm{C}$. En todos los sitios de estudio se evidenció que la temperatura alcanzó los valores más altos durante la época estival, cuando los caudales son mínimos, lo cual queda reflejado en las mediciones de profundidad y ancho de la llanura de inundación, presentando una mayor variabilidad en el río Queuco y menor en el río Quepuca (véase Tabla 1). En este sentido, la variabilidad en la temperatura media diaria del agua puede ser estimada a partir de la temperatura media diaria del aire $(n=311-360$; $p<0.001)$ y las horas de radiación directa en cada sitio de estudio. A este respecto se observó que la temperatura media anual aumentó de 7.53 a $11.87^{\circ} \mathrm{C}$ a medida que disminuía la altitud (de 936 a $370 \mathrm{msnm}$ ). Estas diferencias en variabi-
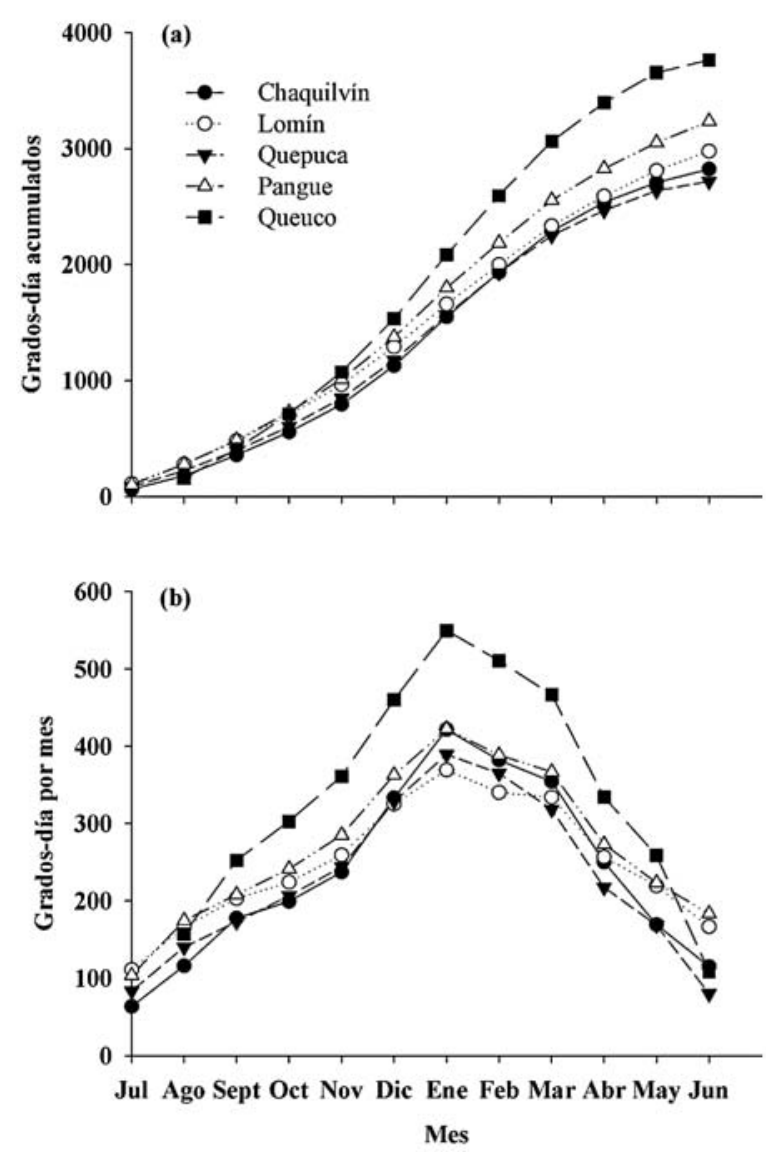

Figura 3. Grados-día acumulados durante el ciclo anual de estudio de julio de 2010 a junio de 2011, (a) grados-día acumulados y (b) grados-día mensuales para cada uno de los ríos andinos. Cumulative degree-days during the annual cycle of the study period, from July 2010 to June 2011, (a) cumulati$v e$ and (b) monthly average degree-days for each of the Andean streams.

lidad espacio-temporal de la temperatura media diaria también se reflejaron en los patrones de grados-día mensual y anual (Figs. 3a y 3b). La variación de grados-día anual registró valores en- 
tre 2716 y 3763 , alcanzando valores más altos a medida que disminuía la altitud. La mayor variabilidad en los patrones de grados-día está en función de las temperaturas de verano (Fig. 3b). Sin embargo, las temperaturas de invierno son importantes para los grados-día en cada sitio, siendo reconocidas por el aumento relativamente lineal entre julio y septiembre.

Los efectos de la estacionalidad sobre la temperatura media diaria y rango medio diario fueron significativos en todos los sitios de estudio $(p<0.001)$. Estas interacciones se condicen con el aumento de la temperatura del aire y una disminución en la profundidad y ancho de la llanura de inundación en época estival. Mientras que la tasa de calentamiento de los ríos fluctuó en primavera desde 0.0624 (río Queuco) a $0.0395{ }^{\circ} \mathrm{C} \mathrm{día}^{-1}$ (río Chaquilvín), y la tasa de enfriamiento de otoño registró valores entre -0.0525 (río Lomín) y $-0.0902{ }^{\circ} \mathrm{C} \mathrm{día}^{-1}$ (río Queuco). En ambos casos las tasas de cambio incrementaron significativamente (modelo de regresión lineal, $p<0.001$ ) con la distancia al origen.

Finalmente, la heterogeneidad térmica de los ríos a mayor altitud respecto al río ubicado a menor altitud se muestra en la figura 4. La diferencia altitudinal en la temperatura media diaria fluctúa entre $0.50-7.65^{\circ} \mathrm{C}$, con una media de $3.15^{\circ} \mathrm{C}$

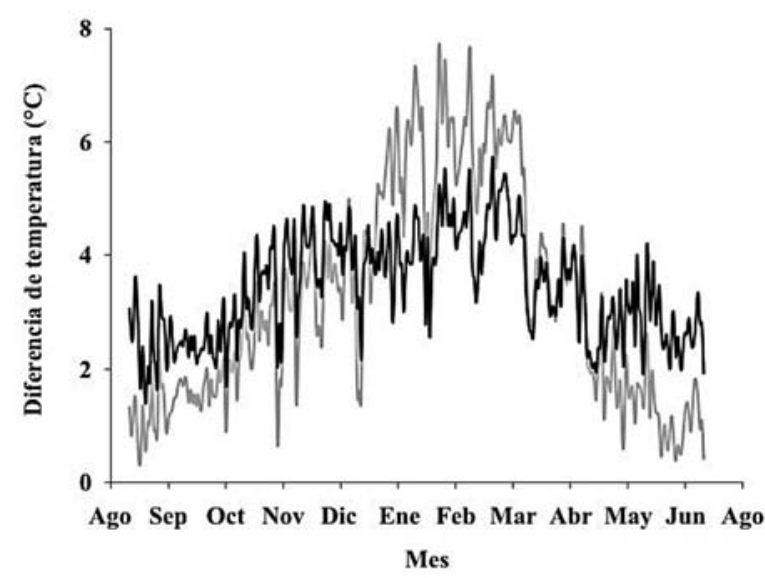

Figura 4. Discontinuidad térmica a través del gradiente altitudinal. Diferencias entre los ríos Queuco-Lomín (línea oscura) y Queco-Chaquilvín (línea clara) durante el período agosto de 2010 a junio de 2011. Thermal discontinuity through altitudinal gradient. Differences among Queuco-Lomín (dark line), and Queco-Chaquilvín (light line) streams from August 2010 to June 2011.
(Queuco-Lomín), y $1.46-5.74{ }^{\circ} \mathrm{C}$, con una media de $3.42{ }^{\circ} \mathrm{C}$ (Queuco-Chaquilvín). En época estival (21 diciembre-21 marzo) se presenta la mayor heterogeneidad térmica con variaciones entre 2.54-7.65 ${ }^{\circ} \mathrm{C}$, con una media de $5.63{ }^{\circ} \mathrm{C}$ (QueucoLomín, y $2.59-5.74{ }^{\circ} \mathrm{C}$, con una media de $4.39^{\circ} \mathrm{C}$ (Queuco-Chaquilvín). En ambos casos la relación fue positiva, debido a que los ríos de altitudes mayores siempre presentaron temperaturas más frías que los tramos más bajos.

\section{DISCUSIÓN}

La variabilidad espacio-temporal de la temperatura es compleja y expresa la influencia local y regional (Uehlinger et al., 2003). En este contexto, la resolución de los registradores de datos (15 min) reveló claras diferencias espaciotemporales en la temperatura del agua de los cinco ríos estudiados. La variabilidad inter-sitio en la temperatura media diaria del agua fue más evidente durante la época estival. Esto se relaciona con los resultados obtenidos por Webb \& Zhang (1997) y Evans et al. (1998) en ríos del Hemisferio Norte, y en Chile Link et al. (2012) para los ríos Vergara e Itata, quienes lo atribuyen a la radiación neta dominada por la entrada de energía solar durante los meses de verano. Esto fue observado en el río Quepuca, que presentó bajas temperaturas, lo cual estaría asociado al desarrollo de una amplia densidad arbórea, abrupta topografía del canal y una baja relación ancho-profundidad. Dichos factores se asocian a la reducción en la cantidad de radiación solar directa y al área disponible para el intercambio de energía desde la atmósfera al río (Hawkins et al., 1997; Webb \& Zhang, 1997; Arscott et al., 2001; Poole \& Berman, 2001; Malcolm et al., 2004). A pesar de que presenta aproximadamente un $43 \%$ de horas con radiación solar directa, la secuencia de sombra generada por el dosel arbóreo y las altas montañas serían los condicionantes a la hora de absorber o reflejar la radiación de onda corta.

Contrariamente, los patrones térmicos encontrados en los ríos Lomín y Chaquilvín responden más a la dinámica meteorológica temporal local, debido a que los sitios se encuentran localiza- 
dos en una zona de valle con escasa secuencia de sombra (matorral y estepa alto-andina), pero con un área disponible mayor para el intercambio de energía (véase en Tabla 1 ancho y profundidad del río), lo cual lleva a que estos sistemas tengan una capacidad térmica relativamente baja. Esta condición concuerda con lo reportado por Isaak \& Hubert (2001), donde el régimen térmico de estos sistemas está más ligado a la radiación solar y a la temperatura del aire. Si bien ambos sistemas se encuentran a una altitud similar, la amplitud térmica entre los sitios es diferente; una situación similar se registró con las temperaturas mínimas y máximas. Estas diferencias inter-sitio se atribuyen a que la relación ancho-profundidad, orientación del río y las horas de radiación directa son distintas, así, Chaquilvín presenta una orientación noreste con un área mayor para intercambio recibiendo en promedio anual $46.6 \%$ de radicación directa, que no solo facilita la cantidad e intensidad de luz que recibe el río durante el día, sino también la capacidad de absorción de energía (Johnson \& Jones, 2000).

Nuestros resultados concuerdan con la relación altitud-temperatura para los hábitats altoandinos de zonas templadas (Nagy \& Grabherr, 2009). Estas diferencias en los patrones térmicos pueden atribuirse primariamente a la altitud y secundariamente a la combinación de distintos factores que se interrelacionan entre sí para influenciar el régimen térmico de estos sistemas fluviales. Así, en el río Queuco, la topografía del canal, la relación ancho-profundidad, la vegetación de ribera, el tipo de sustrato (mayor porcentaje de roca sedimentaria), un mayor aporte de temperatura desde ríos tributarios y el volumen de agua, condicionarían dichos patrones. Mientras que la similitud de los patrones térmicos entre el río Pangue y los ríos de mayor altitud, se asocia a que en su cabecera recibe un aporte importante de alimentación glacial, que estaría determinando la heterogeneidad térmica, debido a la inclusión de temperaturas bajas, en algunos casos cercanas a $0{ }^{\circ} \mathrm{C}$. Sin embargo, se requieren más estudios para estimar la influencia glacial sobre los ríos mediterráneos alto-andinos.

Las fluctuaciones térmicas de invierno no presentaron una variación diaria significativa, pero a mediados de marzo y abril se produce un rápido decaimiento de los patrones térmicos en todos los sitios estudiados; esta disminución se asocia a la intensidad de las primeras lluvias otoñales, que determinaron la naturaleza de la respuesta hidrológica. La respuesta de la temperatura a las condiciones climáticas ha sido reportada como condicionante en la dinámica térmica (Brown et al., 2006). La heterogeneidad en la temperatura del agua entre los sitios de estudio también se ve reflejada en las tasas de cambio y los grados día acumulados durante un ciclo anual; ambos patrones se asocian a la capacidad de buffer de cada río individualmente.

En este trabajo hemos relacionado las diferencias térmicas entre ríos con factores locales como altitud, insolación y presencia de vegetación. A partir de estos datos será necesario a posteriori estimar un balance térmico de cada uno de los ríos considerando datos de caudal e intercambio con el hiporreos; este último puede ser importante como regulador de la temperatura del agua (Evans et al., 1998), y como refugio térmico para los organismos (Acuña \& Tockner, 2009; Wood et al., 2010). Además, los patrones térmicos encontrados podrían ser fuertemente alterados como consecuencia del calentamiento global (Caissie, 2006). En el caso particular de Chile, los registros de temperatura en la zona andina muestran un incremento en la temperatura media diaria del aire de aproximadamente $+0.25^{\circ} \mathrm{C} /$ década entre 1979-2006 (Conama, 2006; Falvey \& Garreaud, 2009). Por lo que un conocimiento detallado de la dinámica de la temperatura de los ríos es esencial para la gestión, protección y conservación futura de los sistemas acuáticos, así como para direccionar la mitigación de los impactos asociados al calentamiento global.

\section{AGRADECIMIENTOS}

Esta investigación agradece a los Proyectos Fondecyt 1095069 y 1108015 la financiación de este estudio, y al Centro de Ciencias Ambientales EULA-Chile de la Universidad de Concepción el soporte logístico prestado. Agradecemos también a la Dra. Nuria Bonada, del Departamento 
de Ecología de la Universidad de Barcelona, y al profesor Fernando Torrejón por sus comentarios sobre el manuscrito, que permitieron mejorar sustancialmente el original.

\section{BIBLIOGRAFÍA}

ACUÑA, V. \& K. TOCKNER. 2009. Surface-subsurface water exchange rates along alluvial river reaches control the thermal patterns in an Alpine river network. Freshwater Biology, 54: 306-320.

ALEXANDER, M. D. \& D. CAISSIE. 2003. Variability and Comparison of Hyporheic Water Temperatures and Seepage Fluxes in a Small Atlantic Salmon Stream1. Ground Water, 41: 72-82.

ARSCOTT, D. B., K. TOCKNER \& J. V. WARD. 2001. Thermal heterogeneity along a braided floodplain river (Tagliamento River, northeastern Italy). Canadian Journal of Fisheries and Aquatic Sciences, 58 (12): 2359-2373.

BERNER, E. K. \& R. A. BERNER. 1996. Global environment: Water, air and geochemical cycles. Prentice Hall, Old Tappan, New Jersey.

BROWN, L. E., D. M. HANNAH \& A. M. MILNER. 2006. Hydroclimatological influences on water column and streambed thermal dynamics in an alpine river system. Journal of Hydrology, 325: 1-20.

BROWN, L. E., D. M. HANNAH \& A. M. MILNER. 2007. Vulnerability of alpine stream biodiversity to shrinking glaciers and snowpacks. Global Change Biology, 13: 958-966.

CAISSIE, D. 2006. The thermal regime of rivers: a review. Freshwater Biology, 51: 1389-1406.

CONAMA. 2006. Estudio de la variabilidad climática en Chile para el siglo XXI. Departamento de Geofísica, Facultad de Ciencias Físicas y Matemáticas, Universidad de Chile, Santiago, Chile.

DALlMAN, P. R. 1998. Plant life in the world's mediterranean climates. University of California Press, Los Angeles, USA.

ERICKSON, T. R. \& H. G. STEFAN. 2000. Linear air-water temperature correlations for streams during open water periods. Journal of Hydrologic Engineering, 5 (3): 317-321.

EVANS, E. C., G. R. MCGREGOR \& G. E. PETTS. 1998. River energy budgets with special reference to river bed processes. Hydrological Processes, 12: 575-595.
FALVEY, M. \& R. D. GARREAUD. 2009. Regional cooling in a warming world: Recent temperature trends in the southeast Pacific and along the west coast of subtropical South America (1979-2006). Journal of Geophysical Research, 114 (D4): 7-14.

GASITH, A. \& V. H. RESH. 1999. Streams in Mediterranean Climate Regions: Abiotic Influences and Biotic Responses to Predictable Seasonal Events. Annual Review of Ecology and Systematics, 30: 51-81.

HAIDEKKER, A. \& D. HERING. 2008. Relationship between benthic insects (Ephemeroptera, Plecoptera, Coleoptera, Trichoptera) and temperature in small and medium-sized streams in Germany: A multivariate study. Aquatic Ecology, 42 (3): 463481.

HAJEK, E. R. 1991. El medio ambiente en Chile. In: La situación ambiental en América Latina. E. R. HAJEK (ed.): 237-294. CIEDLA, Buenos Aires.

HAWKINS, C. P., J. N. HOGUE, L. M. DECKER \& J. W. FEMINELLA. 1997. Channel Morphology, Water Temperature, and Assemblage Structure of Stream Insects. Journal of the North American Benthological Society, 16: 728-749.

HEINO, J. 2002. Concordance of species richness patterns among multiple freshwater taxa: a regional perspective. Biodiversity and Conservation, 11: $137-147$.

IPCC. 2007. Climate Change 2007: Impacts, Adaptation and Vulnerability. Contribution of Working Group II to the Fourth Assessment Report of the Intergovernmental Panel on Climate Change. Cambridge University Press, Cambridge, UK.

ISAAK, D. J. \& W. A. HUBERT. 2001. A hypothesis about factors that affect maximum summer stream temperatures across montane landscapes. Journal of the American Water Resources Association, 37: 351-366.

JACOBSEN, D., R. SCHULTZ \& A. ENCALADA. 1997. Structure and diversity of stream invertebrate assemblages the influence of temperature with altitude and latitude. Freshwater Biology, 38: 247261.

JOHNSON, S. L. 2004. Factors influencing stream temperatures in small streams: substrate effects and a shading experiment. Canadian Journal of Fisheries and Aquatic Sciences, 61: 913-923.

JOHNSON, S. L. \& J. I. JONES. 2000. Stream temperature responses to forest harvest and debris flows in western Cascades Oregon. Canadian 
Journal of Fisheries and Aquatic Sciences, 57: 30-39.

LINK, O., A. HUERTA, A. STEHR, A. MONSALVE, C. MEIER \& M. AGUAYO. 2012. The solarto-stream power ratio: A dimensionless number explaining diel fluctuations of temperature in mesoscale rivers. River Research and Applications: n/a-n/a.

LINK, O., A. MONSALVE, A. STEHR, A. GARCÍA \& R. URRUTIA. 2009. Régimen Térmico del Río Itata. In: La cuenca hidrográfica del río Itata, aportes científicos para su gestión sustentable. $\mathrm{O}$. Parra, J. C. Castilla, H. Romero, R. Quiñones \& A. Camaño (eds.): 44-57. Editorial Universidad de Concepción, Concepción, Chile.

MALCOLM, I. A., D. M. HANNAH, M. J. DONAGHY, C. SOULSBY \& A. F. YOUNGSON. 2004. The influence of riparian woodland on the spatial and temporal variability of stream water temperatures in an upland salmon stream. Hydrology and Earth System Sciences, 8: 449-459.

MALMQVIST, B. \& S. RUNDLE. 2002. Threats to the running water ecosystems of the world. Environmental Conservation, 19: 134-153.

MARDONES, M., E. JAQUE \& D. ZAIO. 1992. Geomorfología de la hoya hidrográfica del río Bio-bío. Instituto Geográfico Militar, Santiago, Chile.

MONSALVE, A., O. LINK \& A. STEHR. 2012. Régimen térmico de ríos: desarrollo, verificación y aplicación de un modelo numérico. Tecnología y Ciencias del Agua, 3: 41-56.

NAGY, L. \& G. GRABHERR. 2009. The Biology of Alpine Habitats. Oxford University Press, New York.

PALMA, A. \& R. FIGUEROA. 2008. Latitudinal diversity of plecoptera (Insecta) on local and global scales. Illiesia, 4: 81-90.

POFF, N. L. \& J. V. WARD. 1990. Physical habitat templates of lotic systems-recovery in the context of historical pattern of spatiotemporal heterogeneity. Environmental Management, 14: 629-645.
POOLE, G. C. \& C. H. BERMAN. 2001. An ecological perspective on in-stream temperature: Natural heat dynamics and mechanisms of human-caused thermal degradation. Environmental Management, 27: 787-802.

ROYER, T. V. \& G. W. MINSHALL. 1997. Temperature patterns in small streams following wildfire. Archiv für Hydrobiologie, 140: 237-242.

SINOKROT, B. A. \& H. G. STEFAN. 1994. Stream water-temperature sensitivy to weather and bed parameters. Journal of Hydraulic Engineering-Asce, 120: 722-736.

TUNG, C. P., T. Y. LEE \& Y. C. YANG. 2006. Modelling climate-change impacts on stream temperature of Formosan landlocked salmon habitat. Hydrological Processes, 20: 1629-1649.

UEHLINGER, U., F. MALARD \& J. V. WARD. 2003. Thermal patterns in the surface waters of a glacial river corridor (Val Roseg, Switzerland). Freshwater Biology, 48: 284-300.

VANNOTE, R. L. \& B. W. SWEENEY. 1980. Geographic Analysis of Thermal Equilibria: A Conceptual Model for Evaluating the Effect of Natural and Modified Thermal Regimes on Aquatic Insect Communities. The American Naturalist, 115: 667695.

WEBB, B. W. 1996. Trends in stream and river temperature. Hydrological Processes, 10: 205-226.

WEBB, B. W., D. M. HANNAH, R. D. MOORE, L. E. BROWN \& F. NOBILIS. 2008. Recent advances in stream and river temperature research. $\mathrm{Hy}$ drological Processes, 22: 902-918.

WEBB, B. W. \& Y. ZHANG. 1997. Spatial and seasonal variability in the components of the river heat budget. Hydrological Processes, 11: 79-101.

WOOD, P. J., A. J. BOULTON, S. LITTLE \& R. STUBBINGTON. 2010. Is the hyporheic zone a refugium for aquatic macroinvertebrates during severe low flow conditions? Fundamental and Applied Limnology/Archiv fur Hydrobiologie, 176: 377-390. 